\title{
A PRIORI TRUTHS AND EMPIRICAL CONFIRMATION
}

\author{
MORRIS LAZEROWITZ \\ ALICE AMBR OSE \\ Smith College
}

C.I. Lewis has distinguished between 'explicity analytic' statements, such as 'All cats are necessarily animals', and 'implicitly analytic' statements, such as 'The class of (existent) cats is included in the class of animals', which affirms that something '(which is necessarily true) is actually true'. ${ }^{1}$ He uses this distinction to justify holding that implicitly analytic statements, which are nevertheless 'genuinely analytic'2, might be established by a procedure comparable to that used to infer empirical generalizations, namely, examination of cases. In his own words: '... that the class of (existent) cats is included in the class of animals can be assured by reference to the meanings of "cat" and "animal" without recourse to further and empirical evidence. But also it might be established -as well established as most laws of science, for example- by generalization from observed instances of cats.' 3 In natural science examination of cases is a means either to rendering a generalization probable or to disestablishing it by exhibiting a counter-instance. Lewis writes that "we may be able to discover that an implicitly analy tic statement, like "All cats are animals", is true by empirical investigation, and without descovering that it is analytic. '4 So what is seemingly implied, if not explicitly stated, in these remarks is the philosophical claim that a logically necessary general statement is open to being rendered probable by an examination of cases. It thus p. 91

1 An Analysis of Knowledge and Valuation. La Salle, Ill: Open Court, 1946,

2 Ibid, p. 93.

3 Ibid.

4 Ibid, p. 93. 
implies that a proposition can be both analytic and true as a matter of fact.

The point of confining the class of cats to the class of existent cats is obscure until we realize that Lewis' claim requires the class of cats to be non-empty. One cannot discover by an empirical investigation that all cats are animals unless cats exist: that is, there is no generalizing from observed instances of cats unless there are cats. Lewis' account is applicable, if indeed it is applicable, to necessarily true general propositions whose subject-class is nonempty. It is inapplicable to such a proposition as All dinosaurs are animals. It becomes clear that a propositions such as All (existent) cats are animals is to be viewed as a conjunction, of which one component is an empirical proposition, in the present example, There are cats. It now becomes unclear how Lewis arrives at the philosophical view that an analytic statement can be established in this manner, for this 'implicitly analytic' statement seems not to be 'genuinely analytic', that is, 'certifiable from examination of meanings', 5 as he claims it is. There is no certifying the existence of cats by analytical means.

As against Lewis, Wittgenstein has stated (Tractatus 6.1222): ' . . logical propositions cannot be confirmed by experience any more than they can be refuted by it. Not only must a proposition of logic be irrefutable by any possible experience, but it must also be unconfirmable by any possible experience'. This characterization can easily be seen to cover any necessarily true proposition $p$, regardless of whether $p$ is of the form $\diamond \sim p$. Although All cats are animals does not assert that all cats necessarily are animals, it is nevertheless analytic, and according to Lewis can be known to be such: by knowing 'the corresponding explicitly analy tic statement. . " that all cats are animals is a logically necessary fact, whose contradiction involves an inconsistency" '.6 If one knows this with regard to any proposition $p$, one know that there is no possibility of there being a counter-instance. To falsify by experience an analytic proposition would be to

5 Ibid.

6 Ibid. 
establish by experience a logically impossible one. That part of Wittgenstein's claim, viz., that a proposition of logic is irrefutable by any possible experience, is thus obvious.

At first glance the other part of Wittgenstein's characterization of logical propositions, namely, that they cannot be confirmed by any theoretical experience, might seem to be in a more questionable position than the claim that they are irrefutable. Within mathematics analogous general propositions -analogous in the sense that they are a priori-can be properly described as having instances on which the generalizations are based. It is proper to speak of supporting cases; at least it must be considered proper if 'supporting case' has a characterizing use in the language of mathematicians. Regarding certain mathematical laws G. Polya wrote that they are 'suggested by special cases, the results being found by induction and proved later'.7 G.H. Hardy described Ramanujan as 'proceeding by induction from numerical examples's which confirm a general conjecture. The process of finding confirming cases of the proposition that any even number is the sum of two primes (e.g., finding that $100=97+3$ ) has been given as an example of collecting 'empirical evidence'.9

What needs to be emphasized here is the vast difference between the uses made of special cases of general propositions in mathematics and in natural science. Special cases in mathematics can serve as a basis for conjecture and thus as a prelude to a demonstration. No mathematician would go about establishing a general proposition by marshalling instances of it. A generalization is not established until a demonstrative proof is at hand. And the latter is an entirely different activity from finding confirming cases. When Lewis speaks of generalizing from observed instances of cats as a relevant procedure for establishing that all cats are animals, it is plain that what he has in mind is intended to parallel the natural scientist's procedure of generalizing from observations which render the

7 How to Solve it. New Jersey: Princeton University Press, 1973, pp. 103-6.

8 Proc. London Mathematical Society (2) XIX (1921), p. 1 viii.

9 R. Lourant and H. Robbins, What is Mathematics? New York: Oxford University Press, 1978, p. 30. 
generalization probable. But in natural science, repeatedly finding confirming instances of a generalization is not a prelude to a demonstrative proof. There is no such thing as a demonstrative proof of a law of nature, i.e., an a priori proof of an empirical proposition. In this respect what is required to make a scientific generalization secure is different in kind from what is required to make a mathematical law secure. A scientific law is never secure in the sense that a counterinstance is unimaginable. To be secure in this sense a scientific law would have to be necessary, i.e., 'genuinely analy tic'.

Looking on All cats are animals as a generalization, and at the same time as analytic, it is clear that no instance of it could fail to be a confirming instance. It is instructive to examine what its conforming instances must be like on Lewis' view that the same inductive procedure used in natural science can establish it. Confirmation would be effected by verifying in the case of each examined cat that it is an animal. That is, observation would establish the truth of each of a set of singular propositions, This cat is an animal, That cat is an animal, etc. There being no discovered counter-instances, one goes on by induction to All cats are animals.

It is obvious that these supporting instances differ in important respects from instances, say, of cats that are mousers. The sentences, 'This cat is an animal' and 'This cat is a mouser', use the demonstrative 'this', whose customary usage is to select particular things from an assemblage. The proposition, This cat is a mouser, implies the possibility of there being cats that are not mousers. But no similar possibility is left open by This cat is an animal. The word 'this' occurring in the expression of the latter is a spurious demonstrative, as it has no selective function. Nor could any such singular proposition be evidence for the supposedly factual generalization. If it could be, then This cat is not an animal would per impossible count as evidence against it. These dissimilarities raise the question whether it is possible for 'empirical investigation' to have any relevance to establishing All cats are animals.

Although the general propositions of number theory are not empirical, something like empirical investigation does 
occur when the truth-value of a given proposition is unknown. The search examples, and counter-examples, parallels in an obvious way the search for examples and counter-examples of natural laws, and the talk surrounding the search is similar. G. Gamov writes concerning Fermat's theorem that for all $n>2, \mathrm{x}^{\mathrm{n}}+\mathrm{y}^{\mathrm{n}} \neq \mathrm{z}^{\mathrm{n}}$, 'the possibility, of course, always remains that the theorem is wrong, and that an example can be found in which the sum of two equal powers of two integers is equal to the same power of the third integer'.10 Entertaining this possibility involves a seeming paradox, for if the theorem is true, no logical possibility of its being 'wrong' exists -there would be no logical possibility of a counter-example. Yet although Gamov's language is such as to make Fermat's theorem analogous to an empirical generalization, one having both truth-values open to it, his language can be so interpreted as to escape objections to which Lewis' treatment of All cats are animals as an empirical generalization is subject.

The difference rests on the character of necessary propositions. We can describe their character indirectly by reference to the sentences expressing them: That a sentence ${ }_{1}$ expresses a necessary proposition is equivalent to the fact that a sentence $_{2}$, to the effect that some related expression in the language in which sentence ${ }_{1}$ occurs has no descriptive use, expresses a verbal truth. E.g., that the sentence ${ }_{1}$ 'All cats are animals' expresses a necessary proposition is equivalent to the fact that the sentence "The expression "cat but not an animal" has no application' 11 expresses a true verbal proposition.12 It is of course clear that it is a fact of language -an empirical fact - that 'cat but not an animal' has no descriptive use. This fact is ascertainable by reference to our language. But inspecting usage in the case of the sentence, 'For all $n>2, x^{n}+y^{n} \neq$ p. 32.

10 One Two Three... Infinity, New York: Bantam Books, 12th printing, 1979,

11 Although the individual words do have application.

12 Alternatively, to the fact that the sentence 2 "The word "animal" applies to everything the word "cat" applies to' expresses a true verbal proposition. For a detailed acount of necessary propositions, see Morris Lazerowitz The Language of Philosophy, Dordrecht, Holland: D. Reidel, 1977, pp. 11-13. It should be clear that it does not state an equivalence between a necessary proposition and the sentence expressing it. 
$\mathrm{z}^{\mathrm{n}}$ ', is of no help -it does not give the information required to cast out of the language of mathematics the expression 'sum of two equal powers of two integers equal to the same power of a third integer'. Proof of Fermat's theorem would have this linguistic result. The paradox of asserting that special cases of $\mathrm{x}^{\mathrm{n}}+\mathrm{y}^{\mathrm{n}} \neq \mathrm{z}^{\mathrm{n}}(n>2)$ make it probable that the inequality holds can be avoided by describing their function in a similar way: as marking it probable that for $n>2$ the expression ' $\mathrm{x}^{\mathrm{n}}+\mathrm{y}^{\mathrm{n}}=\mathrm{z}^{\mathrm{n}}$ ' denotes a sclf-inconsistency, i.e., as making it probable that it has no descriptive place in mathematical language. Probability talk, when directed towards an empirical matter - whether an exprcssion has a use- is paradox free.

By contrast, in the sentence 'All cats are animals' the use of the terms is already known, and amassing instances as a means of establishing the generalization is not construed by Lcwis as making probable that 'cat but not an animal' does, or does not, have a use to refer to somcthing. Hc envisages the empirical investigation of cats as a way of establishing a nonverbal proposition, analogous to inferring a scientific generalization from cases. As is known, in his system of strict implication Lewis demonstrated the two theorems $\sim \diamond p<\sim p$ and $\sim \diamond \sim p<p(18.41$ and 18.42).13 ' . . [F]or any statement asserting something to be logically necessary', he writes, 'thcre is a corresponding implicitly analytic statement, which asserts only that this something is factual'.14 To keep to his illustration, the sccond theorem would be excmplificd by: If all cats necessarily are animals, then it is a matter of fact that all cats are animals. From the consequent of this statement it follows that finding a cat that is not an animal would be a matter of fact impossibility, like finding a cat with three tails. Now a factual impossibility does not eliminate a logical possibility, and in consequence the factual statement, All cats are animals, would leave open the possibility of there being a cat that is not an animal. The term 'cat but not an animal' would denote

13 C. I. Lewis and C, H. Langford, Symbolic Logic. New York: Dover Publications, Inc., 1959, p. 163.

14 An Analysis of knowledge and Valuation, op. cit., p. 92. 
the null class, but could, in principle, denote a membered class. Not only does this go against the Wittgenstein claim that an a priori proposition has no possible refutation; holding that All cats are animals is analytic and at the same time factual implies a contradiction, namely, that it is both impossible and possible for there to be a cat that is not an animal. If analytic, the term 'cat but not an animal' is not linked with any theoretically possible experience, and if factual, it is linked with the nonexistence of a possible experience.

Lewis' position in respect of the consequence it appears to have is like the positivist view that necessary propositions are really verbal. The proposition with which positivists equate an a priori general truth is about usage, and thus empirical, so that the positivist view has been charged with the contradictory consequence that a necessary proposition is not necessary. One cannot reasonably assume that Lewis was unaware of what it is natural to take to be a simple consequence of his view, that an a priori true proposition can be falsified. It is as though he preferred not to see something he knew perfectly well. This calls for an explanation. How could he be aware of the difficulties in his view and fail to admit it is untenable? The explanation must be like that for the positivist, who certainly knew that a necessary proposition is not empirical. His seeming to embrace a contradiction must be thought to have no more substance than an appearance. There is no actual contradiction. We have to think that his claim that an a priori general proposition can be established by observation of instances is not what on the surface it suggests. Lewis represents himself as having made a discovery about a relation between an a priori proposition and an inductive procedure. One possible explanation of how he can appear to do this is that he makes a hidden semantic maneuver in which language is in some way being revised rather than being used to state a fact.

Again and again in philosophy views arc put forward as though they announced some new fact about things or relations. When classical materialists asserted that everything is matcrial they gave the impression of presenting a scientific 
discovery. Yet if one is sensitive to their behavior, for example, their refusal to accept any describable phenomenon as being a counter-instance, coupled with their being unable themselves to describe one, it is easily seen that their account of the nature of phenomena is in no way comparable to the discovery, say, of Brownian motion. What has happened is that one of a pair of antithetical terms, in this case the pair 'material' and 'mental', is artificially deprived of its application, while the other term to all appearances is not. However, the latter remains in the language by academic courtesy, so to speak, for it has in fact lost its former characterizing function. When the term 'mental' is cast out by philosophical fiat, the retained antithetical term loses its use; it no longer functions to distinguish among phenomena.

To return to Lewis' claim that a general statement which has been analytically certified can also be established by an inductive procedure in the way in which a law of physics is established. If knowingly holding a contradictory view is not to be imputed to Lewis, his claim has to be interpreted otherwise. One possible explanation, which helps clear up the paradox, is that Lewis has re-classified an a priori general proposition, in what might be called the spirit of a semantic game, as an inductive generalization. In thc normal use of 'induction', adding confirming instances of a general statement may increase its probability. 15 In such a case as All cats are animals, it is impossiblc for there to be instances which make probable, or increase the probability of, its truth. Examination of instances not only fails to render probable the general proposition, it is a procedure which has no relevance to establishing it. To put it in the vcrbal mode of speech, the expression 'examination of instances' does not refer to a procedure. We cannot, however, suppose that Lewis has misapplied the expression, the use of which he knows perfectly well. One

15 In some cases in which a law of nature is established, it would be ridiculous to suppose that its probability might be increased by doing more tests. E. g., the combination of hidrogen and oxygen to produce water when repeated in a laboratory demonstration does not strengthen the probability of the same result in future cases. 
possible explanation of what has happened is that an innovation in familiar terminology has been introduced: one which stretches the expressions' generalization from observed instances' and 'inductive generalization' to cover a priori propositions. The stretched use is such that 'inductive generalization' is made, by fiat, to apply to all general propositions. Like the use given the term 'material' by the Hobbesian metaphysician, which artificially deprives the term 'mental' of its use in the language, the antithesis of 'inductive generalization' is deprived of its use. The unnoticed consequence of this innovation is that 'inductive generalization', although it ostensibly remains in the language, has lost its use to distinguish among general propositions. If its antithesis, 'noninductive general proposition', is brought back into the language, then Wittgenstein's characterization of an a priori proposition as having no confirmation (as well as no refutation) is preserved. 